\title{
Primary Retroperitoneal Mucinous Tumours Diagnosed in Pregnancy: A Case Report and Literature Review
}

This article was published in the following Dove Press journal: International Journal of Women's Health

\author{
Farshad Tahmasebi $\mathbb{1}^{\prime}$ \\ Mridula Morje ${ }^{2}$ \\ Hina Jamall (iD) \\ Alexander Polson (iD ${ }^{3}$ \\ Nandia Deo (iD ${ }^{\prime}$ \\ 'Obstetrics and Gynaecology, Whipps \\ Cross University Hospital, London, UK; \\ ${ }^{2}$ Obstetrics and Gynaecology, St Thomas' \\ Hospital, London, UK; ${ }^{3}$ Cellular \\ Pathology, Histopathology, St Thomas' \\ Hospital, London, UK
}

Correspondence: Farshad Tahmasebi Flat 20 Charing Cross Mansions, 26 Charing Cross Road, London WC2H ODG, UK

Email tfarshad@hotmail.com

\begin{abstract}
We present the case of a pregnant patient who was found to have a primary retroperitoneal mucinous tumour (PMRT). An abdominal mass measuring $11.5 \times 9.8 \times$ $8.8 \mathrm{~cm}$, initially thought to be of ovarian origin, was found incidentally on ultrasound in a 36-year-old patient at 20 weeks' gestation. Tumour markers were normal. She underwent an elective Caesarean section at 34 weeks, but the uterus, fallopian tubes and ovaries were normal. The mass was found to be retroperitoneal. A clinical decision was made to avoid further surgical exploration at the time. Following further surgical planning, the patient underwent open resection of the retroperitoneal mass, 6 weeks after her Caesarean section. Histology confirmed a moderately differentiated adenocarcinoma suggestive of a primary retroperitoneal mucinous adenocarcinoma. A literature review following this identified five case reports of PMRT in pregnancy in the last decade. In all cases, the patients underwent tumour resection without adverse pregnancy outcomes. While our patient had an uncomplicated pregnancy, she developed a $2 \mathrm{~cm}$ lesion suggestive of disease recurrence 15 months post-operatively. A plan was made for surgical resection. PMRT are rare, but can occur in pregnant patients and patients of childbearing age. Tumour resection should be carried out, but where there is no concrete evidence of tumour invasion, the uterus and ovaries should be spared to avoid infertility and menopause. We would recommend early tumour resection, given its potentially aggressive nature.
\end{abstract}

Keywords: primary retroperitoneal mucinous adenocarcinoma, pregnancy

\section{Introduction}

Primary retroperitoneal mucinous tumours (PRMTs) are rare neoplasms occurring predominantly in women, many of whom are in their reproductive age. ${ }^{1}$ Histologically, PMRTs can be categorised into three main types: mucinous cystadenomas (MC), mucinous borderline tumours or tumours of low malignant potential (MLMP), and mucinous carcinomas (MCa).

Preoperative diagnosis can be difficult to establish as computed tomography (CT) and magnetic resonance imaging (MRI) are unable to determine the exact origin of these tumours or comment on their malignant potential. Because of this and the rare nature of the condition, there are no set guidelines. The diagnosis is usually reached through postoperative histopathological analysis.

In this case report, we describe a patient who was found to have a PMRT during the second trimester of her pregnancy. 


\section{Case Study Details}

A 36-year-old female, gravida 5, para 4, was found to have a right ovarian cyst during an anomaly scan performed at 20 weeks' gestation. The right ovarian cyst measured $11.5 \times 9.8 \times$ $8.8 \mathrm{~cm}$ with a ground-glass appearance with two papillary projections with grade 1 flow on Doppler, the largest of which measured $4.1 \times 3.3 \times 3.1 \mathrm{~cm}$ (Figure 1). The mass was non-tender, and there was no free fluid in the pelvis.

Notably, all tumour markers were within normal range: CA125 31U/mL (0-35), CA19-9 11U/mL (0-35), CEA 2ng/mL (0-4.7). Differential diagnoses included mucinous borderline tumour, ovarian malignancy, and decidualised endometrioma.

The patient underwent an MRI at 20 weeks' gestation (Figure 2), and a repeat scan three weeks later did not show a significant increase in the cyst size.

Following a multidisciplinary team meeting, the patient was offered ultrasonographic monitoring of cyst every 4 weeks, with a preliminary plan to deliver by elective Caesarean section at 34 weeks and perform an oophorectomy at the same time. The cyst size and characteristics remained generally unchanged.

At 34 weeks, the patient underwent an elective Caesarean section. However, both tubes and ovaries appeared normal.

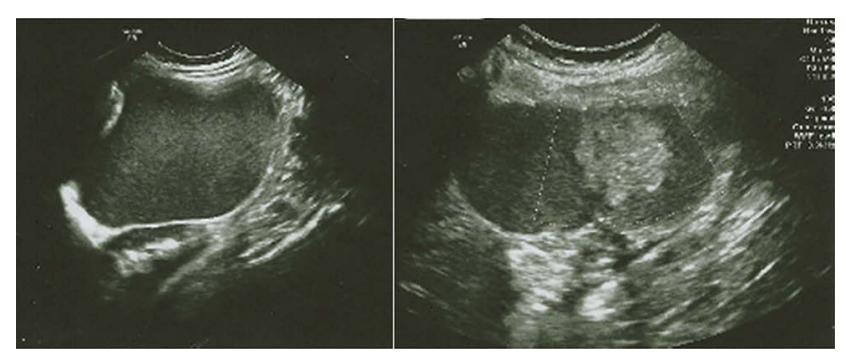

Figure I (Left): TAS at 20 weeks right adnexal mass with ground-glass appearance; (right): Solid component.

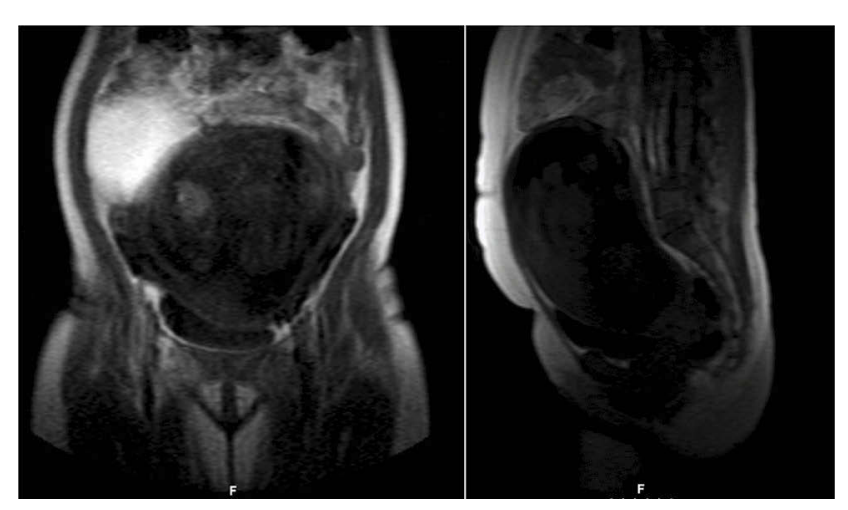

Figure $2 \mathrm{MRI}$ at 20 weeks.
The mass previously observed on imaging appeared to be retroperitoneal, extending to the lower border of the liver. For this reason, no further surgical procedure was carried out at the time.

An MRI done postnatally confirmed that the mass was retroperitoneal in origin (Figure 3 ) and after further surgical planning, the patient underwent a laparotomy, 6 weeks after her Caesarean section. An encapsulated retroperitoneal mass measuring $19 \times 19 \times 18 \mathrm{~cm}$ was removed with no spillage of tumour contents. No other intraabdominal pathology was noted.

Histopathological analysis of the mass revealed a moderately differentiated, intestinal-type adenocarcinoma suggestive of primary retroperitoneal $\mathrm{MCa}$.

She was followed-up by the oncology team. A wholebody fluorodeoxyglucose-positive emission tomography (FDG-PET) was repeated at 1 year, and did not show any evidence of recurrence. All tumour markers remained in the normal range: CA125 25U/mL (0-35), CA19-9 9.7U/mL (0-35), CEA 0.8ng/mL (0-4.7).

However, 15 months later, the patient presented with haematuria and loin-to-groin pain. A CT kidney-uretersbladder showed a $2 \mathrm{~cm}$ lesion in the right paracolic gutter, adjacent to initial site of resection. The lesion lacked FDG uptake, and was suggestive of disease recurrence. Following MDT discussion, surgical intervention was recommended.

\section{Discussion}

PRMT represent $0.1-0.2 \%$ of all neoplasms, and have a male-to-female ratio of $1: 10.1$

A comprehensive meta-analysis conducted by Wolf et al (2017) reported 144 cases of PMRT over a 40-year period (1975-2015). 53\% were $\mathrm{MCa}, 30 \%$ were $\mathrm{MC}$ and $17 \%$



Figure $3 \mathrm{MRI}$ at 6 weeks postpartum. 
were MLMP. The median size of the tumours was 13 $16 \mathrm{~cm}{ }^{1}$

As with our patient, PMRTs may be diagnosed incidentally. However, patients can also present with a palpable abdominal mass or symptoms of mass effect.

As the retroperitoneum is usually devoid of epithelial cells, several theories exist regarding the true origins of these tumours. Their histological similarity to ovarian stroma and the presence of oestrogen receptors in some cases has led to the development of the commonest theory: that the tumours are heterotopic ovarian tissue..$^{2-5}$

However, as demonstrated in our case, heterotopic ovarian tissue is not always found. ${ }^{6}$ The theory of ectopic ovarian tissue is challenged further by the cases diagnosed in men.

A second hypothesis of coelomic metaplasia has gained wide acceptance. It is suggested that clustered deposition of coelomic epithelial cells in the retroperitoneum leads to the development of inclusion cysts which eventually undergo metaplastic changes and acquire a malignant phenotype. ${ }^{7,8}$

Other authors have proposed that the peritoneal mesothelium possesses the potential for Müllerian differentiation, similar to all epithelial ovarian tumours. ${ }^{9}$ This concept has been supported by some authors based on immunohistochemical and electron-microscopic evaluation of the tumours. ${ }^{7}$

Hansmann and Budd dismissed the theory of ovarian histogenesis and proposed that the development of benign retroperitoneal cysts was related to remnants of the embryonal urogenital apparatus in both men and women. The paper determines that majority of the cysts are "pseudomucinous cystadenomas" of mesonephric origin due to the histological presence of hugely varied tissue types including glomeruli and bone. This hypothesis was supported by other authors who reported the presence of Mullerian cysts of the retroperitoneum. ${ }^{10}$

Our case revealed a moderately differentiated adenocarcinoma with intestinal-type differentiation, with the impression of a primary retroperitoneal $\mathrm{MCa}$.

Attaining an accurate pre-operative diagnosis of PMRT is challenging. $\mathrm{MC}$ and $\mathrm{MCa}$ have similar ultrasonographic appearances to each other. ${ }^{1,11} \mathrm{CT}$ and MRI are helpful for surgical planning, but are not effective in excluding malignancy. ${ }^{4,12}$

Additionally, tumour markers such as CA125, CA19-9, CEA, and alpha-fetoprotein rarely aid diagnosis as they are not consistently raised. ${ }^{1,4}$
Wolf et al highlighted that cyst fluid aspiration for preoperative cytology was only diagnostic in $27 \%$ of cases. ${ }^{1}$

Because of the rarity of the tumours, there are no set guidelines on the management or surveillance. All cases reported to date have included total removal of the mass as their main treatment strategy; however, how extensive the surgery remains unclear. ${ }^{5}$ In our case, only the retroperitoneal tumour was removed with sparing of both ovaries and the uterus.

Though specific care is taken to avoid spillage of cyst contents, there is no conclusive evidence linking spillage with increased mortality. Hysterectomy has been associated with reduced recurrence rate, and though salpingooophorectomy and lymph node dissection are often carried out at the same time, they have not been shown to affect survival. $^{13}$

Wolf et al noted that adjuvant chemotherapy was given in $25 \%$ of patients with $\mathrm{MCa}$ with the majority of patients receiving paclitaxel or carboplatin derivatives. ${ }^{1}$ Wolf et al and Myriokefalitaki et al have associated adjuvant chemotherapy with poorer outcomes, and though a few authors recommend chemotherapy in cases where the tumours have invaded adjacent structures, data is limited. ${ }^{1,13}$

Radiotherapy has only been described in the context of bone and peripancreatic metastases. ${ }^{1}$

Whilst 5-year survival for MC and MLMP approaches $100 \%$, the 5 -year survival for MCa is approximately $68 \%{ }^{1}$

Our case involved the additional challenge of pregnancy. Our literature search identified five case reports of PRMT in pregnancy in the last 10 years.

Roma and Malpica describe the case of a patient who presented with pelvic pain at 12 weeks' gestation and was subsequently found to have a $13 \mathrm{~cm}$ multiloculated MLMP. ${ }^{5}$ The imaging modality used for diagnosis, workup and management of the patient is unclear.

Chen et al performed a laparoscopic drainage and resection of a $13 \mathrm{~cm} \mathrm{MC}$ at 14 weeks' gestation. A clinical decision was made to operate at this gestation to reduce the risk of torsion and rupture given the size of the mass. In this case, tocolytic support was used to minimise the risks of pregnancy loss, and the patient went on to have an uncomplicated pregnancy and delivery at 39 weeks. ${ }^{14}$

Kashima et al describe the case of an asymptomatic patient with an incidental finding of a $17 \mathrm{~cm}$ heterogenous retroperitoneal mass on ultrasound at 28 weeks. All tumour markers were within normal range. The mass was removed at laparotomy at 31 weeks' gestation, and histopathology confirmed MCa. The remainder of the pregnancy was uncomplicated, 
and the patient delivered vaginally at 39 weeks' gestation. At 13 months, there was no evidence of recurrence. ${ }^{15}$

Hanhan et al report a similar case where an asymptomatic patient with a $22 \mathrm{~cm}$ retroperitoneal tumour underwent exploratory laparotomy at 30 weeks' gestation, and was found to have an MCa. The CA 19-9 was raised in this patient. Again, the remainder of the pregnancy was uncomplicated, and the patient had a vaginal delivery at 38 weeks. No recurrence was noted at 24 months. ${ }^{16}$

Sonntag et al described an asymptomatic patient with a $5 \mathrm{~cm}$ heterogenous mass inferior to the left kidney which had been found at 30 weeks' gestation and monitored by ultrasound and MRI. Though the CA 19-9 was raised, all other tumour markers were normal. A highly differentiated MCa was removed at Caesarean section at 38 weeks. The patient was offered adjuvant chemotherapy and a staging laparotomy but declined, and was well at her 1 year follow up. There were no adverse outcomes on the pregnancy. ${ }^{17}$

Our case emphasises the challenges of imaging and managing PMRTs during pregnancy. Unlike the five reported cases, our patient underwent fertility-sparing tumour resection at 6 weeks postpartum. Unfortunately, she developed disease recurrence at 15 months. A case can be made for a need for more aggressive management of these tumours, with a focus on early resection.

\section{Conclusion}

In conclusion, PRMT is rare and difficult to diagnose preoperatively. No clear consensus has been met regarding the origins of these tumours. Tumour resection is the mainstay of treatment. The role of adjuvant chemotherapy continues to be unclear. Close follow-up and appropriate management based on histological appearance are crucial in these patients so that recurrence can be identified and treated at an early stage.

\section{Consent}

Written informed consent has been provided by the patient to have the case details and any accompanying images published. No institutional approval is needed to publish the case.

\section{Acknowledgment}

The abstract of this paper "Primary retroperitoneal mucinous tumours diagnosed in pregnancy: a case report and literature review" was presented at the 27th World Congress on Ultrasound in Obstetrics and Gynaecology as a poster presentation/conference talk with interim findings. The poster's abstract was published in "Poster Abstracts" in Journal of Ultrasound in Obstetrics and Gynaecology.

\section{Disclosure}

The authors report no conflicts of interest in this work.

\section{References}

1. Wolf B, Kunert C, Horn L, Einenkel J. Management of primary retroperitoneal mucinous tumors: a retrospective meta-analysis. Int J Gynecol Cancer. 2017;27:1064-1071.

2. Hiroshi I. A case of retroperitoneal mucinous cystadenocarcinoma thought to be derived from ectopic ovary. Cancer Chemother. 2015;42(10):1265-1267.

3. Acharya SR, Dasgupta P, Das S, Halder S, Panda N. Retropancreatic ovarian tumor. Indian J Surg. 2016;78(3):232-234. doi:10.1007/ s12262-015-1387-4

4. Paraskevakou H, Orfanos S, Diamantis T, Konstantinidou A, Patsouris E. Primary retroperitoneal mucinous cystadenoma. A rare case with two cysts and review of the literature. Hippokratia. 2014;18 (3):278-281.

5. Roma AA, Malpica A. Primary retroperitoneal mucinous tumors: a clinicopathologic study of 18 cases. Am J Surg Pathol. 2009;33:526-533. doi:10.1097/PAS.0b013e3181909018

6. Roth LM, Ehrlich CE. Mucinous cystadenocarcinoma of the retroperitoneum. Obstet Gynecol. 1977;49:486-488.

7. Balat O, Aydin A, Sirikci A, et al. Huge primary mucinous cystadenoma of the retroperitoneum mimicking a left ovarian tumor. Eur J Gynaecol Oncol. 2001;22:454-455.

8. Banerjee R, Gough J. Cystic mucinous tumours of the mesentery and retroperitoneum: report of three cases. Histopathology. 1988;12:527-532. doi:10.1111/his.1988.12.issue-5

9. Isse K, Harada K, Suzuki Y, et al. Retroperitoneal mucinous cystadenoma: report of two cases and review of the literature. Pathol Int. 2004;54:132-138. doi:10.1111/pin.2004.54.issue-2

10. Hansmann GH, Budd JW. Massive unattached retroperitoneal tumours. Am J Pathol. 1931;1931(7):631-673.

11. Manrai M, Takesita N, Ishida H, et al. Primary retroperitoneal mucinous cystic tumors with borderline malignancy: a case report and literature review. Clini and Pract. 2015;5(722):14-16. doi:10.4081/ cp. 2015.722

12. Knezevic S, Ignjatovic I, Lukic S, et al. Primary retroperitoneal mucinous cystadenoma: a case report. World $J$ Gastroenterol. 2015;21(7):5427-5431. doi:10.3748/wjg.v21.i17.5427

13. Myriokefalitaki E, Lugman I, Potdar N, Brown L, Steward W, Moss EL. Primary retroperitoneal mucinous cystadenocarcinoma (PMRCa): a systematic review of the literature and meta-analysis. Arch Gynaecol Obstet. 2016;293(4):709-720. doi:10.1007/s00404015-3975-8

14. Chen C, Chiu L, Lin J, Liu W. Pelvic retroperitoneal cyst during pregnancy. Taiwan J Obstet Gynecol. 2013;52:117-119. doi:10.1016/ j.tjog.2012.07.041

15. Kashima K, Yahata T, Fujita K, Tanaka K. Primary retroperitoneal mucinous cystadenocarcinoma associated with pregnancy. Int J Gynecol Cancer. 2008;18(5):908-912. doi:10.1111/j.15251438.2007.01130.x

16. Hanhan HM, Gungorduk K, Ozdemir IA, et al. Primary retroperitoneal mucinous cystadenocarcinoma during pregnancy. J Obstet Gynaecol (Lahore). 2014;34(6):535-538. doi:10.3109/ 01443615.2014 .910501

17. Sonntag B, Lelle RJ, Steinhard J, Brinkmann OA, Hungermann D, Kiesel L. Retroperitoneal mucinous adenocarcinoma occurring during pregnancy in a supernumerary ovary. $J$ Obstet Gynaecol (Lahore). 2009;25(5):515-516. doi:10.1080/01443610500193478 
International Journal of Women's Health

Dovepress

\section{Publish your work in this journal}

The International Journal of Women's Health is an international, peerreviewed open-access journal publishing original research, reports, editorials, reviews and commentaries on all aspects of women's healthcare including gynecology, obstetrics, and breast cancer. The manuscript management system is completely online and includes a very quick and fair peer-review system, which is all easy to use. Visit http://www.dovepress.com/testimonials.php to read real quotes from published authors.

Submit your manuscript here: https://www.dovepress.com/international-journal-of-womens-health-journal 